\title{
Interannual variation in the metazoan parasite communities of bigeye trevally Caranx sexfasciatus (Pisces, Carangidae)
}

\author{
Juan Violante-González ${ }^{1,2, *}$, Scott Monks ${ }^{3, *}$, Yesenia Gallegos-Navarro ${ }^{2}$, Nataly G. Santos-Bustos ${ }^{2}$, \\ Princessa J. Villalba-Vasquez ${ }^{2}$, Jesús G. Padilla-Serrato ${ }^{4}$, and Griselda Pulido-Flores ${ }^{3}$ \\ ${ }^{1}$ Facultad de Ecología Marina, Universidad Autónoma de Guerrero, 39390 Acapulco, Mexico \\ ${ }^{2}$ Centro de Ciencias de Desarrollo Regional, Universidad Autónoma de Guerrero, 39630 Acapulco, Mexico \\ ${ }^{3}$ Centro de Investigaciones Biológicas, Universidad Autónoma del Estado de Hidalgo, 42000 Pachuca, Mexico \\ ${ }^{4}$ Conacyt - Facultad de Ecología Marina, Universidad Autónoma de Guerrero, 39390 Acapulco, Mexico
}

Received 9 September 2019, Accepted 6 January 2020, Published online 31 January 2020

\begin{abstract}
Parasite communities in Caranx sexfasciatus were characterized and analyzed to determine any interannual variations in structure and/or species composition. In total, 422 C. sexfasciatus were collected from Acapulco Bay, Mexico, between May 2016 and March 2019. Thirty-two taxa of metazoan parasites were identified: five Monogenea, thirteen Digenea, one Acanthocephala, one Cestoda, three Nematoda, seven Copepoda, and two Isopoda. Monogeneans were the most frequent and abundant parasite species in all sampling years. Parasite species richness at the component community level varied significantly from 8 (May 2016) to 25 (March 2019) and was similar to previous reports for other species of Carangidae. The component communities and infracommunities in C. sexfasciatus were characterized by low parasite species numbers, low diversity, and dominance of a single species (the monogenean Neomicrocotyle pacifica). Parasite community structure and species composition varied between sampling years and climatic seasons. Seasonal or local fluctuations in some biotic and abiotic environmental factors probably explain these variations.
\end{abstract}

Key words: Parasites, Marine fish, Caranx sexfasciatus, Acapulco, México.

\begin{abstract}
Résumé - Variation interannuelle des communautés de parasites métazoaires de la carangue à gros yeux Caranx sexfasciatus (Pisces, Carangidae). Les communautés de parasites de Caranx sexfasciatus ont été caractérisées et analysées afin de déterminer si elles connaissent des variations interannuelles dans la structure et/ou la composition des espèces. Au total, 422 C. sexfasciatus ont été collectés dans la baie d'Acapulco, au Mexique, entre mai 2016 et mars 2019. Trente-deux taxons de parasites métazoaires ont été identifiés : cinq Monogenea, treize Digenea, un Acanthocephala, un Cestoda, trois Nematoda, sept Copepoda et deux Isopoda. Les monogènes étaient les espèces de parasites les plus fréquentes et les plus abondantes au cours de toutes les années d'échantillonnage. La richesse en espèces de parasites au niveau de la communauté des composants variait considérablement de 8 (mai 2016) à 25 (mars 2019) et était similaire aux rapports précédents pour d'autres espèces de Carangidae. Les communautés et infracommunautés de $C$. sexfasciatus étaient caractérisées par un faible nombre d'espèces de parasites, une faible diversité et la dominance d'une seule espèce (le monogène Neomicrocotyle pacifica). La structure des communautés de parasites et la composition des espèces variaient entre les années d'échantillonnage et les saisons climatiques. Les fluctuations saisonnières ou locales de certains facteurs environnementaux biotiques et abiotiques expliquent probablement ces variations.
\end{abstract}

\section{Introduction}

Parasite communities in marine fish consist of ectoparasites (monogeneans, copepods, and isopods) and endoparasites (digeneans, cestodes, nematodes, and acanthocephalans). Both types of parasite exhibit different transmission strategies to infect their hosts; for example, ectoparasites are often transmitted

\footnotetext{
*Corresponding authors: monks. scott@gmail. com;
} viojuang@yahoo.com.mx directly between individual hosts through contact, whereas endoparasites often use trophic transmission routes [9, 26, 37]. Transmission strategies of parasites are linked to host behavior. For instance, ectoparasite populations may be more abundant on species of fish that form large schools than on solitary species $[23,29,31]$, because the probability of a transmission stage (e.g., eggs, larvae) coming into contact with a host increases as host density rises.

Studies of parasite communities are conducted mainly at two different hierarchical levels: component community 
(between locations or host populations) and infracommunity (between individual hosts) $[14,17,51]$. In recent years, a central theme in parasite ecology has been the identification of the factors (biotic or abiotic) determining species richness and composition in these communities. However, an understanding is also needed of how consistent parasite community richness and species composition are in space and time. For example, spatial or temporal variation in community structure may indicate how important local environmental factors (abiotic and biotic conditions) are in structuring a parasite community, particularly when disparities are found between different host species [1, 24, 46, 50, 53]. There is still no consensus on whether marine parasite communities can exhibit temporal variations in structure and species composition. Some studies suggest that the infection levels of most parasite species do not experience significant changes over time, and that communities tend to be generally stable in species composition for long periods of time $[4,12,16,43]$. Others indicate that even though parasite species composition may be relatively stable over time, a community can undergo substantial changes in structure due to variations in local environmental factors [14, 39, 51].

The bigeye trevally, Caranx sexfasciatus Quoy \& Gaimard 1825 , is an economically important pelagic fish species distributed widely along the eastern coast of the Pacific Ocean from CA, USA to Ecuador and the Galapagos Islands [2]. Bigeye trevally often form large stationary daytime schools, but usually tend to be solitary at night when feeding. Their diet consists mainly of fish, but the species also preys on squids and crustaceans [2]. Despite its ecological and economic importance, no information is available to date on its parasite fauna along the Pacific coast of Mexico. The present study objectives were (1) to characterize the metazoan parasite communities of C. sexfasciatus; and (2) to evaluate possible interannual variability in its parasite communities.

\section{Materials and methods}

A total of 422 individuals of $C$. sexfasciatus were obtained from commercial fishermen over a four-year period (May 2016-March 2019) from Acapulco Bay (16 $51^{\prime} \mathrm{N} ; 99^{\circ}$ $\left.52^{\prime} \mathrm{W}\right)$ in Guerrero, Mexico. The tropical Pacific region experiences two distinct climatic seasons: a rainy period from June to November (total precipitation $\approx 950 \mathrm{~mm}$ ), and a dry period from December to May (total precipitation $<70 \mathrm{~mm}$ ). In the years 2017 and 2019, a single sampling was carried out, but in a different climatic season: August (rainy season) and March (dry season), respectively. In 2016 and 2017, two samplings were carried out per year, which included both climatic seasons: May and October in 2016, and May and November in 2018. Records of surface temperatures of the ocean waters and salinity per sampling date were obtained from other studies carried out at the same location but not yet published. Multivariate El Niño index values (MEI) for each sampling date were obtained from the National Oceanic and Atmospheric Administration (NOAA: https://www.esrl.noaa.gov/psd/enso/mei/table.html). Fish were measured and weighed at the time of collection. A complete necropsy was made for each specimen and all parasites were collected from the internal and external organs [52].
Contents of the digestive tract were examined to identify prey items consumed by this species. Dietary items were identified to the family level when possible. Prey item analysis was carried out using the frequency of occurrence method [19, 30]. Parasites were identified to the lowest possible taxonomic level and vouchers of the most abundant and best-preserved specimens deposited in the Coleccion Nacional de Helmintos (CNHE), Instituto de Biologia, Universidad Nacional Autonoma de Mexico, Mexico City.

Infection levels for each parasite species were described using prevalence (percent of fish infected with a particular parasite species); mean abundance (mean number of individual parasites of a particular species per examined fish), expressed as the mean \pm standard deviation $(S D)$; and intensity (number of a particular parasite species per infected fish), expressed as range (minimum-maximum) $[5,6,8]$. Possible differences in infection levels between sampling years and climatic seasons were identified using $G$-tests [44], and a general linear model (GLM) for abundance. The dispersion index (DI = variance to mean abundance ratio) was applied to describe parasite dispersion patterns. The infracommunity index (ICI) [55], which describes the frequency of double and multiple infections by a single species of parasite in a distinct host (affinity level of a species of parasite; i.e. species with great tendency to join the infracommunity), was also calculated. Spearman's correlation coefficient $\left(r_{s}\right)$ was used to determine possible relationships between total host length and abundance or DI values of each parasite species.

Analyses were done at the levels of component community (i.e. total parasite species in all fish collected at a sampling year) and infracommunity (i.e. total parasite species in each individual fish). Component community parameters included total species richness, total number of individuals of each parasite species, the Shannon-Wiener Index $(H)$ as a measure of diversity, species evenness (equitability), and the Berger-Parker Index (BPI) as a measure of numerical dominance [25]. The qualitative Sorensen index and quantitative percentage of similarity (PS) index were used to evaluate similarity and difference in parasite community species composition between sampling dates. Differences between component community parameters were identified with Student $t$ and $\chi^{2}$ tests.

Infracommunities were described in terms of mean number of parasite species per host, mean number of individuals of each species, and the mean Brillouin Diversity Index $\left(H^{\prime}\right)$ value per host. The multivariate general linear model (GLM) was used to identify possible differences in infracommunity parameters (dependent variables) between sampling years and climatic seasons (predictor variables); fish body size (total length) was used as a covariate to control for the influence of host body size. The significance of all statistical analyses was established at $p<0.05$, unless stated otherwise. A multivariate analysis (principal component analysis, PCA) was applied to identify factors that influenced parasite infracommunity species richness and diversity. The predictor variables used were: Fulton's condition factor $\left(K_{n}\right)$ [13]; host diet diversity (calculated as the diversity of items consumed by host populations at each sampled date, through the use of the Shannon-Wiener index at the family level); number of endoparasite and ectoparasite species; surface temperature; salinity; MEI values; sampling year; and climatic 
season. The Kaiser-Meyer-Olkin (KMO) test of sampling adequacy for each variable in the model, as well as the Bartlett sphericity test, which evaluates the possibility that there is redundancy among variables were applied. The variance maximizing rotation method was applied to produce the two ordination axes. Discriminant function analyses based on Mahalanobis distances were used to identify possible differences in parasite community structure between sampling years. The probability of correct classification expected by chance alone of fishes to any of the four sampling years was calculated using the proportional chance criterion, which is a simple method to account for differences in sample sizes between the groups being compared [36]. Only parasite species with a prevalence $>10 \%$ in at least one of the sampling years (a component species; sensu [7]) were included in this analysis.

\section{Results}

\section{Species composition}

Thirty-two taxa of metazoan parasites (23 of helminths and nine of Crustacea) were recovered and identified (9866 individual parasites) from 422 individuals of $C$. sexfasciatus collected from Acapulco Bay, Mexico. Five species of Monogenea (adults), 13 Digenea (12 adults and one metacercaria), one Acanthocephala (adult), three Nematoda (one adult and two larvae), seven Copepoda, and two species of Isopoda were collected (Table 1). Species richness was highest among the digeneans, representing $37.1 \%$ of the total species, followed by copepods $(20 \%)$. Based on infection site, 14 species of parasite were classified as ectoparasites and 18 as endoparasites. The numbers of species of ectoparasite varied significantly from $2.02 \pm 0.90$ in 2016 to $2.41 \pm 1.20$ in 2019 (ANOVA $F_{3,405}=3.23, p<0.05$ ) (Fig. 1). Eleven species, Neomicrocotyle pacifica Yamaguti 1968, Protomicrocotyle manteri Bravo-Hollis 1966, Pseudomazocraes selene Hargis 1957, Bucephalus varicus Manter 1940, Ectenurus virgulus Looss 1910, Synaptobothrium apharei Yamaguti 1970, Tetraphyllidea gen. sp., Anisakis sp., Caligus alalongae Krøyer 1863, Ca. robustus Bassett-Smith 1898, and Lernanthropus ilishae Chin 1948 occurred in the parasite communities of $C$. sexfasciatus in all sampling years (Table 1).

\section{Interannual variation in infection levels}

For eight of these perennial species (the exceptions were Pr. manteri, S. apharei and Ca. robustus), prevalence varied significantly between sampling years, though no clear pattern was observed in variation. For the parasites $B$. varicus $(G=35.1, p<0.05)$ and $E$. virgulus $(G=12.9, p<0.05)$ infection percentages were highest in May 2016. For the Tetraphyllidea cestode, prevalence was highest during October 2016. The species $N$. pacifica $(G=21.8, p<0.05)$, Anisakis sp. $(G=12.1$, $p<0.05)$ and Ca. alalongae $(G=42.3, p<0.05)$ exhibited their highest percentages of infection in 2017. For $L$. ilishae $(G=17.1, p<0.05)$, the highest percentage occurred in May 2018, while for Ps. selene $(G=34.9, p<0.05)$, it was in November 2018 (Table 1).
In contrast to the percentage infection results, only five species ( $N$. pacifica $\left[\mathrm{GLM} ; F_{3,406}=7.92, p<0.05\right]$, Ps. selene [GLM; $\left.F_{3,406}=3.05, p<0.05\right]$, B. varicus $\left[\mathrm{GLM} ; F_{3,406}=3.10\right.$, $p<0.05]$, Tetraphyllidea cestode [GLM; $F_{3,406}=5.49$, $p<0.05$ ], and Ca. alalongae [GLM; $F_{3,406}=5.31$, $p<0.05])$ exhibited significant interannual variation in abundance. Abundance for B. varicus, Ps. selene and Tetraphyllidea larvae was highest in October 2016. For $\mathrm{Ca}$. alalongae, it was highest in 2017, while for N. pacifica it was highest in 2019 (Table 1). Prevalence values were generally positively correlated with mean abundance values, indicating that the most prevalent species also were the most abundant $\left(r_{s}=0.946\right.$, $p<0.01)$.

\section{Spatial dispersion}

The dispersion index (DI) values indicated that at least 10 species $(31 \%)$ exhibited an aggregated dispersion pattern in one or more sampling years (Table 1). Higher mean aggregation values (DI $>8$ ) occurred in several years for the monogeneans $N$. pacifica and Pr. manteri (Table 1). The DI values of each parasite species correlated positively with its prevalence $\left(r_{s}=0.824, p<0.01\right)$, total number of individuals $\left(r_{s}=0.878, p<0.01\right)$, and mean abundance $\left(r_{s}=0.871\right.$, $p<0.01)$ values, but not with host body size or sample size $(p>0.05)$.

\section{Host diet composition}

The alimentary spectrum of the fish included twelve prey items, of which smaller fish (36\%), crab larvae $(24.5 \%)$ and penaeid larvae $(15.6 \%)$ represented the largest proportions. Additonal prey items accounted for $24 \%$ of the diet, and included amphipods, polychaetes, mollusk larvae, isopods, stomatopods, pistol crabs, and copepods. Diet composition varied between sampling years $(t=3.10, p<0.05)$. For example, in May 2016 diet composition was less varied $(H=0.68$, Table 2), with crab larvae being the main prey item. Feeding habits also changed with fish body size; larger individuals consumed higher percentages of fish while smaller ones fed on higher percentages of crustaceans and other prey.

\section{Component community}

Species richness of parasite by sampling year (Table 2) varied widely from eight (May 2016) to 25 (March 2019) $\left(\chi^{2}=11.6, p<0.05\right)$. No correlation was observed between sample size and species richness at this level, indicating that the different sample sizes used in the analyses had no effect on the results. The total number of individual parasites ranged from 670 (October 2016) to 3465 (March 2019) $\left(\chi^{2}=3,997.2\right.$, $p<0.05)$. The only dominant species was the monogenean $N$. pacifica (Table 2), although its degree of dominance varied significantly between sampling years $(t=4.70, p<0.01)$. Shannon-Wiener diversity index values were generally low, ranging from 0.90 (October 2016) to 1.40 (March 2019), but varied between sampling years $(t=3.68, p<0.01)$. Similarity between component communities was slightly low overall 
Table 1. Parasite infection parameters for Caranx sexfasciatus from Acapulco Bay, Mexico.

\begin{tabular}{|c|c|c|c|c|c|c|c|c|c|c|}
\hline Parasite taxa & $\begin{array}{l}\text { Site on } \\
\text { host }\end{array}$ & $\begin{array}{c}\text { Accession } \\
\text { number }\end{array}$ & Year & $n^{\mathrm{b}}$ & $\begin{array}{l}\text { Prevalence } \\
(\%)\end{array}$ & Total $^{\mathrm{c}}$ & Abundance $^{\mathrm{d}}$ & $\begin{array}{l}\text { Range of } \\
\text { intensity }^{\mathrm{e}}\end{array}$ & $\mathrm{DI}^{\mathrm{f}}$ & $\mathrm{ICI}^{\mathrm{g}}$ \\
\hline \multicolumn{11}{|l|}{ Digenea } \\
\hline \multirow{3}{*}{$\begin{array}{l}\text { Bucephalus margaritae Ozaki \& } \\
\text { Ishibashi, } 1934\end{array}$} & \multirow[t]{3}{*}{ Intestine } & \multirow[t]{3}{*}{10,997} & 2017 & 97 & 2.06 & 3 & $0.03 \pm 0.71$ & $1-2$ & 0.33 & 0.007 \\
\hline & & & $2018_{M}$ & 63 & 4.76 & 3 & 0.05 & $0-1$ & & 0.021 \\
\hline & & & 2019 & 110 & 3.64 & 10 & $0.09 \pm 2.4$ & $1-6$ & 2.27 & 0.015 \\
\hline \multirow[t]{6}{*}{ Bucephalus varicus ${ }^{\mathrm{h}}$ Manter, 1940} & \multirow[t]{6}{*}{ Intestine } & \multirow[t]{6}{*}{10,998} & $2016_{M}$ & 65 & $40 *$ & 81 & $1.25 \pm 2.2$ & $1-9$ & 1.52 & 0.146 \\
\hline & & & $2016_{\mathrm{O}}$ & 36 & 27.78 & 67 & $1.86 \pm 7.4^{*}$ & $1-25$ & 8.13 & 0.109 \\
\hline & & & 2017 & 97 & 24.74 & 70 & $0.72 \pm 3$ & $1-12$ & 3.04 & 0.086 \\
\hline & & & $2018_{M}$ & 63 & 14.29 & 26 & $0.41 \pm 2.8$ & $1-9$ & 2.72 & 0.064 \\
\hline & & & $2018_{\mathrm{N}}$ & 51 & 3.92 & 2 & 0.04 & $0-1$ & & 0.016 \\
\hline & & & 2019 & 110 & 19.09 & 157 & $1.43 \pm 8.6$ & $1-31$ & 9.89 & 0.081 \\
\hline Neoapocreadium cf. caranxi & Intestine & & 2017 & 97 & 1.03 & 1 & 0.01 & $0-1$ & & 0.004 \\
\hline \multirow[t]{3}{*}{ Didymozoid larvae } & \multirow[t]{3}{*}{ Intestine } & & 2017 & 97 & 4.12 & 6 & $0.06 \pm 0.6$ & $1-2$ & 0.22 & 0.014 \\
\hline & & & $2018_{N}$ & 51 & 1.96 & 2 & 0.04 & $0-2$ & & 0.008 \\
\hline & & & 2019 & 110 & 2.73 & 3 & 0.03 & $0-1$ & & 0.012 \\
\hline \multirow[t]{5}{*}{ Ectenurus virgulus ${ }^{\mathrm{h}}$ Looss, 1910} & \multirow[t]{5}{*}{ Intestine } & 11,000 & $2016_{M}$ & 65 & $29.23 *$ & 38 & $0.58 \pm 3$ & $1-14$ & 4.39 & 0.107 \\
\hline & & 11,001 & $2016_{O}$ & 36 & 25.00 & 35 & $0.97 \pm 3.8$ & $1-13$ & 3.69 & 0.098 \\
\hline & & & 2017 & 97 & 20.62 & 32 & $0.33 \pm 1.1$ & $1-5$ & 0.75 & 0.071 \\
\hline & & & $2018_{\mathrm{N}}$ & 51 & 9.80 & 10 & $0.20 \pm 1.7$ & $1-5$ & 1.50 & 0.041 \\
\hline & & & 2019 & 110 & 13.64 & 38 & $0.35 \pm 3.2$ & $1-14$ & 4.11 & 0.058 \\
\hline Hirudinella ventricosa (Pallas, 1774) & \multirow[t]{3}{*}{ Intestine } & & 2017 & 97 & 5.15 & 5 & 0.05 & $0-1$ & & 0.018 \\
\hline \multirow[t]{2}{*}{ Baird, 1853} & & & $2018_{\mathrm{N}}$ & 51 & 1.96 & 1 & 0.02 & $0-1$ & & 0.008 \\
\hline & & & 2019 & 110 & 0.91 & 1 & 0.01 & $0-1$ & & 0.004 \\
\hline $\begin{array}{l}\text { Lecithocladium excisum (Rudolphi, } \\
\text { 1819) Lühe, } 1901\end{array}$ & Intestine & & 2019 & 110 & 1.82 & 3 & $0.03 \pm 0.7$ & $1-2$ & 0.33 & 0.008 \\
\hline \multicolumn{3}{|c|}{ 1940) Caballero \& Caballero, 1950} & $2018_{N}$ & 51 & 1.96 & 1 & 0.02 & $0-1$ & & 0.008 \\
\hline Mecoderus oligoplitis Manter, 1940 & Intestine & & 2019 & 110 & 0.91 & 1 & 0.01 & $0-1$ & & 0.004 \\
\hline $\begin{array}{l}\text { Pseudopecoeloides carangi } \\
\text { (Yamaguti, 1938) Yamaguti, } 1940\end{array}$ & Intestine & 11,002 & $2018_{M}$ & 63 & 1.59 & 1 & 0.02 & $0-1$ & & 0.007 \\
\hline Stephanostomum ditrematis & Intestine & & $2018_{N}$ & 51 & 1.96 & 1 & 0.02 & $0-1$ & & 0.008 \\
\hline (Yamaguti, 1939) Manter, 1947 & & & 2019 & 110 & 1.82 & 2 & 0.02 & $0-1$ & & 0.008 \\
\hline $\begin{array}{l}\text { Stephanostomum carangis } \\
\text { (Yamaguti, 1951) Caballero, } 1952\end{array}$ & Intestine & & 2017 & 97 & 1.03 & 1 & 0.01 & $0-1$ & & 0.004 \\
\hline Synaptobothrium apharei (Yamaguti, & Intestine & 11,004 & $2016_{O}$ & 36 & 2.78 & 2 & 0.06 & $0-2$ & & 0.011 \\
\hline 1970) Leon-Regagnon, Perez-Ponce & & 11,005 & 2017 & 97 & 4.12 & 8 & $0.08 \pm 1.4$ & $1-4$ & 1 & 0.014 \\
\hline de Leon \& Lamothe-Argumedo, & & & $2018_{N}$ & 51 & 7.84 & 6 & $0.12 \pm 1$ & $1-3$ & 0.67 & 0.033 \\
\hline 1997 & & & 2019 & 110 & 5.45 & 7 & $0.06 \pm 0.4$ & $1-2$ & 0.14 & 0.023 \\
\hline Monogenea & & & & & & & & & & \\
\hline $\begin{array}{l}\text { Heteromicrocotyla carangis } \\
\text { Yamaguti, } 1953\end{array}$ & Gills & & 2019 & 110 & 0.91 & 1 & 0.01 & $0-1$ & & 0.004 \\
\hline $\begin{array}{l}\text { Neobenedenia melleni (MacCallum, } \\
\text { 1927) Yamaguti, } 1963\end{array}$ & Gills & & 2019 & 110 & 0.91 & 1 & 0.01 & $0-1$ & & 0.004 \\
\hline Neomicrocotyle pacifica ${ }^{\mathrm{h}}$ (Meserve, & Gills & 10,992 & $2016_{M}$ & 65 & 86.15 & 899 & $13.83 \pm 15.5$ & $1-57$ & 15.06 & 0.314 \\
\hline 1938) Yamaguti, 1968 & & 10,993 & $2016_{O}$ & 36 & 44.44 & 262 & $7.28 \pm 21.2$ & $2-76$ & 27.19 & 0.174 \\
\hline & & & 2017 & 97 & $91.75^{*}$ & 2271 & $23.41 \pm 19.5$ & $1-103$ & 14.88 & 0.317 \\
\hline & & & $2018_{M}$ & 63 & 85.71 & 589 & $9.35 \pm 10.9$ & $1-61$ & 10.85 & 0.386 \\
\hline & & & $2018_{N}$ & 51 & 62.75 & 718 & $14.08 \pm 39.1$ & $1-184$ & 67.98 & 0.260 \\
\hline & & & 2019 & 110 & 80.91 & 2818 & $25.62 \pm 31.4^{*}$ & $1-142$ & 31.10 & 0.343 \\
\hline Protomicrocotyle manteri ${ }^{\mathrm{h}}$ Bravo- & Gills & 10,994 & $2016_{M}$ & 65 & 63.08 & 199 & $3.06 \pm 5.1$ & $1-20$ & 5.26 & 0.230 \\
\hline Hollis, 1966 & & 10,995 & $2016_{O}$ & 36 & 47.22 & 229 & $6.36 \pm 18.3$ & $1-76$ & 24.98 & 0.184 \\
\hline & & & 2017 & 97 & 45.36 & 114 & $1.18 \pm 2.6$ & $1-13$ & 2.66 & 0.157 \\
\hline & & & $2018_{M}$ & 63 & 46.03 & 67 & $1.06 \pm 1.5$ & $1-6$ & 1.02 & 0.207 \\
\hline & & & $2018_{\mathrm{N}}$ & 51 & 56.86 & 173 & $3.39 \pm 8.4$ & $1-45$ & 11.82 & 0.236 \\
\hline & & & 2019 & 110 & 60.00 & 265 & $2.41 \pm 4.3$ & $1-24$ & 4.62 & 0.254 \\
\hline Pseudomazocraes selene ${ }^{\mathrm{h}}$ Hargis, & Gills & 10,996 & $2016_{M}$ & 65 & 1.54 & 1 & 0.02 & $0-1$ & & 0.006 \\
\hline 1957 & & & $2016_{O}$ & 36 & 13.89 & 13 & $0.36 \pm 3.1^{*}$ & $1-8$ & 3.58 & 0.054 \\
\hline & & & 2017 & 97 & 1.03 & 1 & 0.01 & $0-1$ & & 0.004 \\
\hline & & & $2018_{M}$ & 63 & 3.17 & 9 & $0.14 \pm 3.5$ & $1-7$ & 2.78 & 0.014 \\
\hline & & & $2018_{\mathrm{N}}$ & 51 & $19.61 *$ & 16 & $0.31 \pm 1$ & $1-4$ & 0.58 & 0.081 \\
\hline & & & 2019 & 110 & 12.73 & 19 & $0.17 \pm 0.5$ & $1-2$ & 0.18 & 0.054 \\
\hline
\end{tabular}


Table 1. (Continued)

\begin{tabular}{|c|c|c|c|c|c|c|c|c|c|c|}
\hline Parasite taxa & $\begin{array}{c}\text { Site on } \\
\text { host }\end{array}$ & $\begin{array}{c}\text { Accession } \\
\text { number }^{\mathrm{a}}\end{array}$ & Year & $n^{\mathrm{b}}$ & $\begin{array}{c}\text { Prevalence } \\
(\%)\end{array}$ & Total $^{\mathrm{c}}$ & Abundance $^{\mathrm{d}}$ & $\begin{array}{l}\text { Range of } \\
\text { intensity }^{\mathrm{e}}\end{array}$ & $\mathrm{DI}^{\mathrm{f}}$ & $\mathrm{ICI}^{\mathrm{g}}$ \\
\hline \multicolumn{11}{|l|}{ Cestoda } \\
\hline \multirow[t]{4}{*}{ Tetraphyllidea gen. sp. ${ }^{\text {h }}$} & Intestine & & $2016_{O}$ & 36 & $16.67 *$ & 18 & $0.50 \pm 2.7$ & $1-8$ & 2.40 & 0.065 \\
\hline & & & 2017 & 97 & 3.09 & 3 & 0.03 & $0-1$ & & 0.011 \\
\hline & & & $2018_{N}$ & 51 & 7.84 & 8 & $0.16 \pm 0.8$ & $1-3$ & 0.33 & 0.033 \\
\hline & & & 2019 & 110 & 0.91 & 1 & 0.01 & $0-1$ & & 0.004 \\
\hline \multicolumn{11}{|l|}{ Acanthocephala } \\
\hline \multirow[t]{2}{*}{ Rhadinorhynchus sp. } & Intestine & & $2018_{N}$ & 51 & 1.96 & 7 & 0.14 & $0-7$ & & 0.008 \\
\hline & & & 2019 & 110 & 1.82 & 2 & 0.02 & $0-1$ & & 0.008 \\
\hline \multicolumn{11}{|l|}{ Nematoda } \\
\hline \multirow[t]{5}{*}{ Anisakis sp. ${ }^{\mathrm{h}}$ larvae } & Mesentery & & $2016_{M}$ & 65 & 4.62 & 6 & $0.09 \pm 1.7$ & $1-4$ & 1.50 & 0.017 \\
\hline & & & $2016_{O}$ & 36 & 5.56 & 3 & $0.08 \pm 0.7$ & $1-2$ & 0.33 & 0.022 \\
\hline & & & 2017 & 97 & $16.49 *$ & 31 & $0.32 \pm 1.4$ & $1-6$ & 1.06 & 0.057 \\
\hline & & & $2018_{M}$ & 63 & 6.35 & 4 & 0.06 & $0-1$ & & 0.029 \\
\hline & & & 2019 & 110 & 6.36 & 10 & $0.09 \pm 0.8$ & $1-3$ & 0.43 & 0.027 \\
\hline Contracaecum sp. larvae & Mesentery & & $2018_{N}$ & 51 & 1.96 & 1 & 0.02 & $0-1$ & & 0.008 \\
\hline Procamallanus sp. & Intestine & & 2019 & 110 & 0.90 & 1 & 0.01 & $0-1$ & & 0.004 \\
\hline \multicolumn{11}{|l|}{ Copepoda } \\
\hline Bomolochus sp. & Gills & 123 & $2016_{O}$ & 36 & 2.78 & 1 & 0.03 & $0-1$ & & 0.011 \\
\hline \multirow{6}{*}{$\begin{array}{l}\text { Caligus alalongae }{ }^{\mathrm{h}} \text { Krøyer, } \\
1863\end{array}$} & Gills & 120 & $2016_{M}$ & 65 & 9.23 & 6 & 0.09 & $0-1$ & & 0.034 \\
\hline & & & $2016_{O}$ & 36 & 8.33 & 3 & 0.08 & $0-1$ & & 0.033 \\
\hline & & & 2017 & 97 & $22.68 *$ & 54 & $0.56 \pm 1.8^{*}$ & $1-7$ & 1.39 & 0.078 \\
\hline & & & $2018_{M}$ & 63 & 3.17 & 2 & 0.03 & $0-1$ & & 0.014 \\
\hline & & & $2018_{N}$ & 51 & 1.96 & 1 & 0.02 & $0-1$ & & 0.008 \\
\hline & & & 2019 & 110 & 0.91 & 1 & 0.01 & $0-1$ & & 0.004 \\
\hline \multirow{8}{*}{$\begin{array}{l}\text { Caligus chorinemi Krøyer, } \\
1863 \\
\text { Caligus robustus }{ }^{\mathrm{h}} \text { Bassett- } \\
\text { Smith, } 1898\end{array}$} & Gills & 121 & 2017 & 97 & 2.06 & 2 & 0.02 & $0-1$ & & 0.007 \\
\hline & & & 2019 & 110 & 8.18 & 10 & $0.09 \pm 0.3$ & $1-2$ & 0.10 & 0.035 \\
\hline & Gills & 122 & $2016_{M}$ & 65 & 40.00 & 41 & $0.63 \pm 0.7$ & $1-4$ & 0.31 & 0.146 \\
\hline & & & $2016_{O}$ & 36 & 44.44 & 30 & $0.83 \pm 1.3$ & $1-5$ & 0.84 & 0.174 \\
\hline & & & 2017 & 97 & 32.99 & 49 & $0.51 \pm 0.6$ & $1-3$ & 0.21 & 0.114 \\
\hline & & & $2018_{M}$ & 63 & 38.10 & 51 & $0.81 \pm 1.8$ & $1-7$ & 1.49 & 0.172 \\
\hline & & & $2018_{N}$ & 51 & 49.02 & 44 & $0.86 \pm 1.2$ & $1-5$ & 0.77 & 0.203 \\
\hline & & & 2019 & 110 & 50.91 & 89 & $0.81 \pm 0.8$ & $1-4$ & 0.36 & 0.216 \\
\hline $\begin{array}{l}\text { Caligus mutabilis Wilson, } \\
1905\end{array}$ & Gills & & 2017 & 97 & 1.03 & 1 & 0.01 & $0-1$ & & 0.004 \\
\hline \multirow[t]{3}{*}{ Ergasilus sp. } & Gills & & $2016_{O}$ & 36 & 8.33 & 4 & $0.11 \pm 0.6$ & $1-2$ & 0.25 & 0.033 \\
\hline & & & $2018_{N}$ & 51 & 5.88 & 3 & 0.06 & $0-1$ & & 0.024 \\
\hline & & & 2019 & 110 & 1.82 & 2 & 0.02 & $0-1$ & & 0.008 \\
\hline Lernanthropus ilishae $^{\mathrm{h}}$ Chin, & Gills & 124 & $2016_{O}$ & 36 & 5.56 & 2 & 0.06 & $0-1$ & & 0.022 \\
\hline \multirow{4}{*}{1948} & & & 2017 & 97 & 9.28 & 11 & $0.11 \pm 0.7$ & $1-3$ & 0.36 & 0.032 \\
\hline & & & $2018_{M}$ & 63 & $17.46^{*}$ & 13 & $0.21 \pm 0.4$ & $1-2$ & 0.14 & 0.079 \\
\hline & & & $2018_{N}$ & 51 & 1.96 & 1 & 0.02 & $0-1$ & & 0.008 \\
\hline & & & 2019 & 110 & 15.45 & 19 & $0.17 \pm 0.3$ & $1-2$ & 0.10 & 0.065 \\
\hline \multicolumn{11}{|l|}{ Isopoda } \\
\hline \multirow[t]{2}{*}{ Gnathia sp. } & Gills & & 2019 & 110 & 2.73 & 3 & 0.03 & $0-1$ & & 0.012 \\
\hline & & & $2018_{M}$ & 63 & 1.59 & 36 & 0.57 & $0-36$ & & 0.007 \\
\hline Rocinella signata Schioedte \& & Gills & 125 & $2016_{O}$ & 36 & 2.78 & 1 & 0.03 & $0-1$ & & 0.011 \\
\hline \multirow[t]{2}{*}{ Meinert, 1879} & & & $2018_{N}$ & 51 & 1.96 & 1 & 0.02 & $0-1$ & & 0.008 \\
\hline & & & 2019 & 110 & 0.91 & 1 & 0.01 & $0-1$ & & 0.004 \\
\hline
\end{tabular}

${ }^{\text {a }}$ Specimens deposited at the CNHE (Colección Nacional de Helmintos, Instituto de Biología, Universidad Nacional Autónoma de México).

${ }^{\mathrm{b}}$ Number of fish examined.

${ }^{c}$ Total number of individual parasites collected.

${ }^{\mathrm{d}}$ Number of parasites per fish (mean $\pm \mathrm{SD}$ ).

${ }^{\mathrm{e}}$ Minimum to maximum number of parasites present.

${ }^{\mathrm{f}}$ Variance to mean ratio.

Infracommunity index.

${ }^{\mathrm{h}}$ Component species.

Subscripts to the right of the sampling year indicate the sampling month: $\mathrm{M}=\mathrm{May}, \mathrm{O}=$ October, $\mathrm{N}=$ November.

*Significantly different measurements of prevalence $(G$-test) and abundance (two-way ANOVA) $(p<0.05)$. 


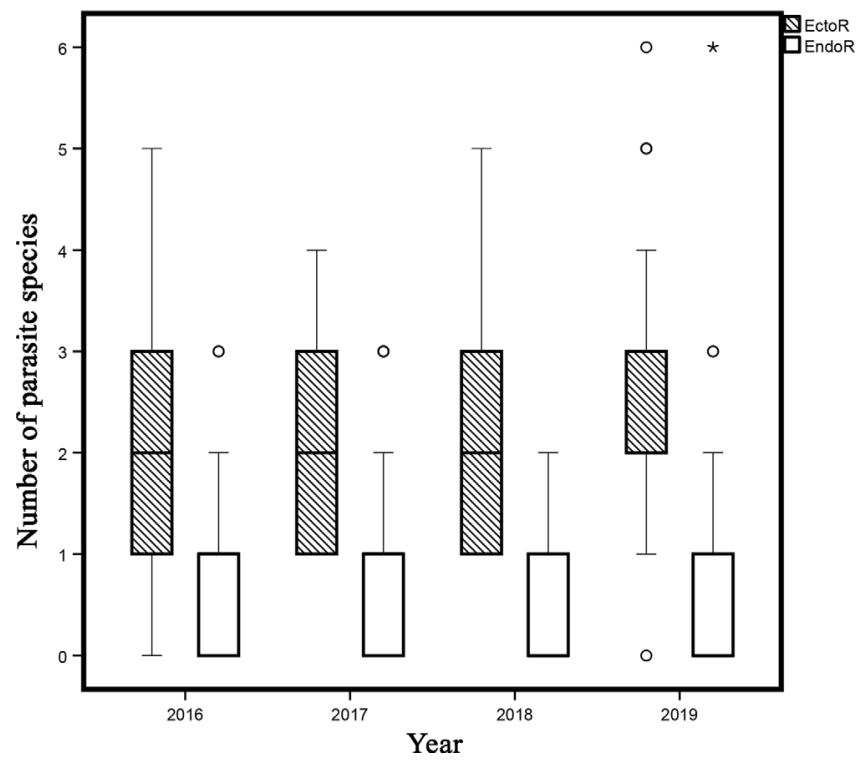

Figure 1. Box plot of species number of ectoparasites (monogenean and crustacean), and endoparasites (larvae and adults) in Caranx sexfasciatus from Acapulco Bay. The bottom and top of the boxes represent the lower and upper quartiles respectively; the median is shown as a line through the centre of the boxes; outliers are indicated with circles for ectoparasites and asterisks for endoparasites.

( $<65 \%$, Fig. 2) and was higher at the qualitative level $($ mean $=64.05 \%)$ than at the quantitative level $($ mean $=56.15 \%)$.

\section{Infracommunity}

The body size of individuals of $C$. sexfasciatus varied from $19.0 \pm 1.3 \mathrm{~cm}$ (October 2016) to $28.8 \pm 4.5 \mathrm{~cm}$ (August 2017) (Table 2), and it differed significantly between sampling years (ANOVA $F_{3,402}=32.3, p<0.01$ ). Mean species richness of parasites ranged from $2.22 \pm 1.30$ to $2.96 \pm 1.54$, and the mean number of individual parasites per fish from $12.71 \pm 11.5$ to $31.50 \pm 30.35$ (Table 2). The Brillouin diversity index $\left(H^{\prime}\right)$ values varied from $0.53 \pm 0.48$ to $0.71 \pm 0.58$. The mean number of species $\left(\mathrm{GLM} ; F_{3,406}=3.95, p<0.01\right)$ and the mean number of individuals (GLM; $F_{3,406}=10.72, p<0.01$ ) were highest in March 2019 (Table 2), although mean diversity $\left(H^{\prime}\right)$ did not exhibit significant variation between sampling years $(p>0.05)$. Dominance of the monogenean $N$. pacifica at the infracommunity level was very similar to that at the component level (Table 2). Considering all samples, the mean number of species $\left(r_{s}=-0.368, p<0.01\right)$, and the mean diversity $\left(r_{s}=-0.098, p<0.05\right)$ exhibited negative correlations with host body size of fish, while the mean number of individual parasites was positively correlated $\left(r_{s}=0.313, p<0.01\right)$. The infracommunity index values (ICI) indicated that $N$. pacifica, Pr. manteri, and Ca. robustus had the highest number of double or multiple co-occurrences with other parasite species (ICI mean $>0.15$ ) in all the sampling years (Table 1).

\section{Multivariate analyses}

The KMO (0.476) and the Bartlett's tests results $\left(\chi^{2}=2,944.2 ; d f=45, p<0.001\right)$ for the PCA, applied to identify the influence of biotic and abiotic factors on parasite infracommunity structure (Fig. 3), indicated that there is a sufficient relationship between the number of variables studied and the sample sizes, confirming the relevance of the PCA. The first three component variables generated by the model explained $61.9 \%$ of total variance, contributing $24.9 \%$ (eigenvalue $=3.74$ ), $19.8 \%$ (eigenvalue $=2.97$ ), and $17.2 \%$ (eigenvalue $=2.58$ ). The first variable suggests that the richest and most diverse infracommunities among all the sampled years were characterized by a significant number of ectoparasite and endoparasite component species, with more homogeneous species abundances. The second variable indicated that both the total number of ectoparasites and the parasite load per infracommunity were highest in larger hosts with a more varied diet. The third variable associated host diet with the environmental factors that were studied (Table 3). This variable indicated that host diet was more varied during the dry season when water surface temperature was slightly cooler (as suggested by the negative correlations), which occurred mainly during the final sampling year (Tables 2 and 3).

Table 2. Characteristics of the parasite component communities and infracommunities of Caranx sexfasciatus from Acapulco Bay, Mexico.

\begin{tabular}{|c|c|c|c|c|c|c|c|c|c|c|c|c|c|c|c|}
\hline \multirow[t]{2}{*}{ Year } & \multirow[t]{2}{*}{ Date } & \multirow[t]{2}{*}{$\mathrm{SST}{ }^{\circ} \mathrm{C}$} & \multirow{2}{*}{$\begin{array}{c}\text { Salinity } \\
\text { (ppt) }\end{array}$} & \multirow{2}{*}{$\begin{array}{l}\text { No. } \\
\text { fish }\end{array}$} & \multirow{2}{*}{$\begin{array}{l}\text { Length } \\
(\mathrm{cm})\end{array}$} & \multirow[t]{2}{*}{ Diet } & \multicolumn{6}{|c|}{ Component communities } & \multicolumn{3}{|c|}{ Infracommunities } \\
\hline & & & & & & & $\begin{array}{l}\text { Species } \\
\text { richness }\end{array}$ & $\begin{array}{c}\text { No. of } \\
\text { parasites }\end{array}$ & BPI & $\begin{array}{c}\text { Dominant } \\
\text { species }\end{array}$ & $\begin{array}{l}\% \text { of } \\
\text { dom. } \\
\text { infra. }\end{array}$ & $H$ & $\begin{array}{c}\text { Mean } \\
\text { number } \\
\text { of species }\end{array}$ & $\begin{array}{c}\text { Mean } \\
\text { number } \\
\text { of parasites }\end{array}$ & $\begin{array}{c}\text { Mean value } \\
\text { of Brillouin } \\
\text { Index }\end{array}$ \\
\hline $2016 a$ & May $^{\mathrm{d}}$ & 28 & 34.9 & 65 & $23.9 \pm 4.5$ & 0.68 & 8 & 1271 & 0.707 & Neom & 73.8 & 0.90 & $2.70 \pm 1.10$ & $19.5 \pm 16.79$ & $0.70 \pm 0.41$ \\
\hline $2016 b$ & $\mathrm{Oct}^{\mathrm{r}}$ & 30 & 34.4 & 36 & $19.0 \pm 1.3$ & 0.90 & 14 & 670 & 0.391 & Neom & 13.9 & 1.15 & $2.56 \pm 1.83$ & $18.61 \pm 27.86$ & $0.71 \pm 0.58 *$ \\
\hline 2017 & Aug $^{r}$ & 30 & 34.6 & 97 & $28.8 \pm 4.5$ & 0.96 & 18 & 2663 & 0.853 & Neom & 88.7 & 1.25 & $2.89 \pm 1.30$ & $27.4 \pm 20.1 .37$ & $0.56 \pm 0.43$ \\
\hline $2018 \mathrm{a}$ & May $^{\mathrm{d}}$ & 29 & 34.8 & 63 & $26.7 \pm 3.1$ & 0.70 & 11 & 801 & 0.735 & Neom & 74.6 & 1.04 & $2.22 \pm 1.30$ & $12.71 \pm 11.50$ & $0.53 \pm 0.48$ \\
\hline $2018 b$ & Nov $^{r}$ & 30 & 33.6 & 51 & $24.0 \pm 3.5$ & 1.0 & 18 & 996 & 0.721 & Neom & 47.0 & 1.25 & $2.41 \pm 1.25$ & $19.53 \pm 33.98$ & $0.64 \pm 0.44$ \\
\hline 2019 & $\operatorname{Mar}^{\mathrm{d}}$ & 28 & 34.1 & 110 & $26.5 \pm 5.9$ & 1.1 & 25 & 3465 & 0.813 & Neom & 64.5 & 1.40 & $2.96 \pm 1.54^{*}$ & $31.50 \pm 30.35^{*}$ & $0.61 \pm 0.49$ \\
\hline
\end{tabular}

SST $=$ sea surface temperature, Length $=$ total length, Diet $=$ diet variety, BPI $=$ Berger-Parker Index; $\%$ of dom. infra $=$ percentage of dominated infracommunities, $\mathrm{H}=$ Shannon-Wiener diversity index, Neom = Neomicrocotyle pacifica.

* Significant at $p<0.05$. Letters as superscripts represent codes of assignment ranges of locations and climatic seasons $(\mathrm{d}=\mathrm{dry}, \mathrm{r}=$ rainy) used in the multivariate statistical analysis. 

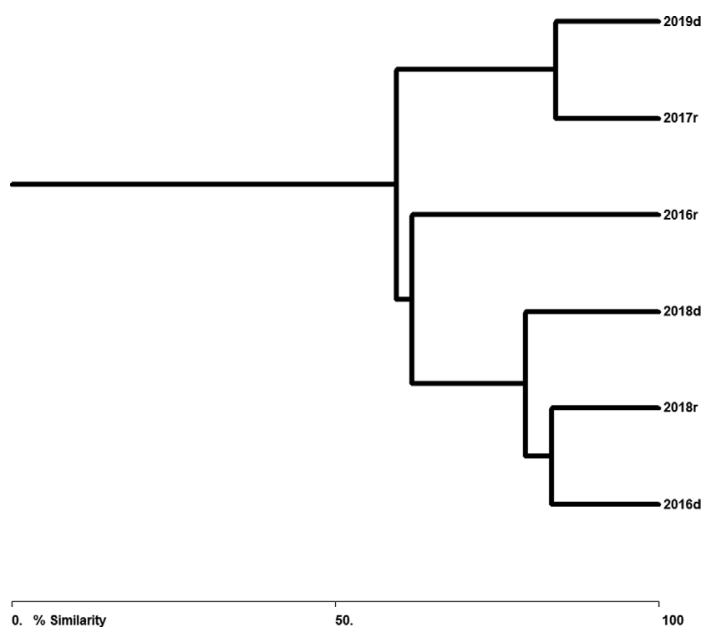

Figure 2. Similarity percentages (Bray-Curtis index) for parasite communities of Caranx sexfasciatus between sampling years. $\mathrm{d}=$ dry season, $\mathrm{r}=$ rainy season.

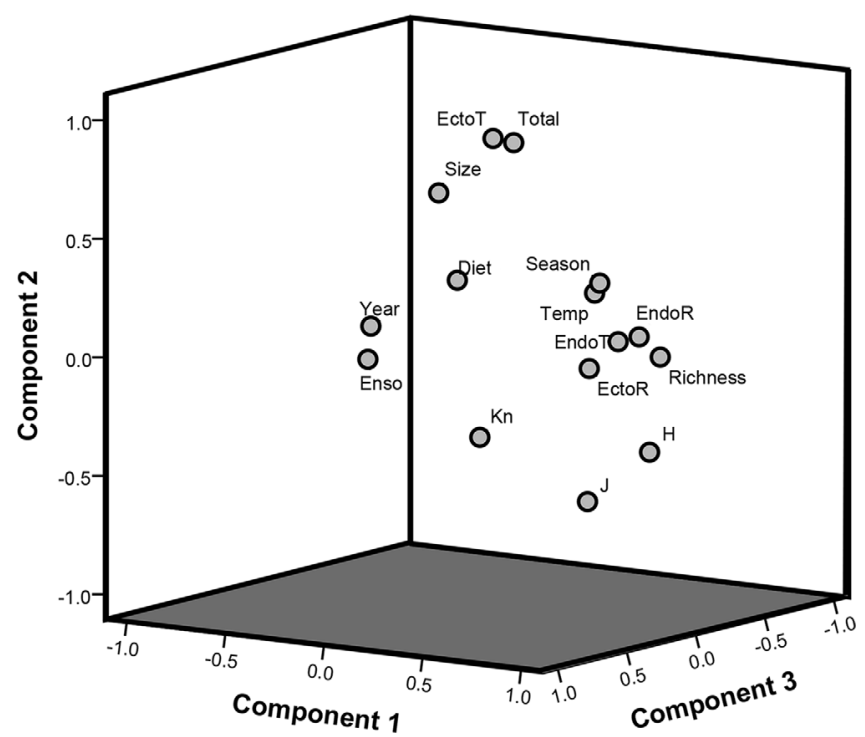

Figure 3. Scatter plot of the principal component analysis (PCA) of factors that influence the species richness and diversity of the parasite infracommunities of Caranx sexfasciatus, from Acapulco Bay. Predictor variables: Year $=$ sampling year, Season $=$ climatic season (dry/rainy), Temp = surface temperature values, Enso = Multivariate El Niño index, Size = host body size (total length), $K_{n}=$ condition factor, Diet $=$ host diet variety, EndoR = number of endoparasite species, EctoR = number of ectoparasite species, EndoT $=$ total number of endoparasites per host, EctoT $=$ total number of ectoparasites per host. Infracommunity parameters: Richness $=$ number of parasite species per infracommunity, Diversity $=$ Brillouin diversity index values, Load $=$ total number of parasites per infracommunity, Evenness = species evenness.

In the model constructed to identify possible interannual differences in parasite community structure, the first two discriminant variables explained $86.9 \%$ of the total variance, contributing $56.2 \%$ (eigenvalue $=0.164$ ) and $30.6 \%$ (eigenvalue $=0.090$ ). A significant overall group effect was observed (Wilks' lambda $=0.759, p<0.001$ ). Individual fish were mainly distributed along the first axis (Fig. 4). Dimensionality tests showed that the four sampling years were significantly different in both dimensions $\left(\chi^{2}=110.37, d f=15\right.$, $p<0.001)$. Each fish was correctly assigned to one of the four sampling years with a $41.1 \%$ accuracy, more than double that achieved by chance alone $(16 \%)$.

Of the nine component species (i.e. prevalence $>10 \%$ ), only five were accepted by the model (Table 4) based on their lower Wilks' lambda values. High abundance of the monogenean Pr. manteri and lower abundance of the digenean E. virgulus were characteristic of hosts collected in 2016. The monogenean $N$. pacifica and the copepod L. ilishae effectively functioned to identify hosts that were collected in 2019, while the copepods $C a$. alalongae and L. ilishae effectively distinguished between hosts collected in 2017 and 2018.

\section{Discussion}

\section{Parasite community species composition}

All the present parasite species records (23 helminths and nine crustaceans) are new geographical records for $C$. sexfasciatus on the Pacific coast of Mexico (Table 1). Ectoparasites (five monogeneans, seven copepods, and two isopods) numerically dominated the parasite communities, representing $92.71 \%$ of the total number of individual parasites recovered. This predominance is probably due to the monogeneans' transmission strategies (direct contact), increasing their transmission probabilities especially in gregarious fish such as $C$. sexfasciatus $[27,42]$. Eight of the reported monogenean species (including N. pacifica, Pr. manteri, and Ps. selene) have been recorded in Carangidae from the Pacific coast of Mexico [32]. Copepods are a highly diverse group that mainly parasitize marine fish $[22,28,45]$; however, this crustacean group has received limited attention in Mexico [14]; in the present study, they were the second most diverse group and represented $22 \%$ of the total taxa recovered (Table 1).

\section{Interannual variation in parasite infection levels}

At least five species of parasite (two endoparasites $B$. varicus and a tetraphyllidean cestode, and three ectoparasites - N. pacifica, Ps. selene and Ca. alalongae) exhibited significant interannual differences in infection levels (Table 1). Endoparasites often are trophically transmitted, so variations in infection levels can be explained by interannual changes in host diet and availability of prey harboring infective stages $[16,18,23,38]$. The population density and gregarious behavior (schooling behavior) of C. sexfasciatus [2] may be a better explanation for the annual variation observed in the three species of ectoparasites. Fish that form large schools facilitate ectoparasite transmission, particularly parasites with a direct transmission cycle such as monogeneans and copepods [14, 23, 39, 41, 51].

\section{Dispersion pattern}

The DI values indicated that at least $31 \%$ of the species of parasites exhibited aggregate dispersion in one or more of the sampling years (Table 1). Aggregation is considered a typical 
Table 3. Summary of the principal component analysis (PCA) on biotic and abiotic factors that influence the diversity and species richness of the infracommunities of Caranx sexfasciatus. The three principal component axes are indicated by PC1, PC2 and PC3, respectively. Variables that contribute to the total explained variance in each PC are in bold. Eigenvalues, percentage of variance, and accumulative variance are shown at the end of the table. Communality $=$ total influence of the variable with respect to all other associated variables. Uniqueness = percentage of variability that is not predicted by the variable in the model.

\begin{tabular}{|c|c|c|c|c|c|}
\hline & & & & Communality & Uniqueness \\
\hline Variables & $\mathrm{PC} 1$ & $\mathrm{PC} 2$ & $\mathrm{PC} 3$ & $R^{2}$ & $\%$ not predicted \\
\hline Richness & 0.961 & 0.050 & 0.045 & 0.928 & 0.072 \\
\hline Diversity & 0.892 & -0.355 & 0.024 & 0.922 & 0.078 \\
\hline EctoR & 0.709 & 0.005 & 0.200 & 0.543 & 0.457 \\
\hline EndoR & 0.705 & 0.085 & -0.167 & 0.532 & 0.468 \\
\hline EndoT & 0.667 & 0.075 & -0.070 & 0.455 & 0.545 \\
\hline Evenness & 0.594 & -0.590 & 0.048 & 0.703 & 0.297 \\
\hline EctoT & 0.148 & 0.904 & 0.095 & 0.848 & 0.152 \\
\hline Load & 0.244 & 0.894 & 0.082 & 0.865 & 0.135 \\
\hline Size & -0.171 & 0.633 & 0.033 & 0.431 & 0.569 \\
\hline$K_{n}$ & 0.123 & -0.351 & 0.156 & 0.162 & 0.838 \\
\hline Diet & 0.135 & $\mathbf{0 . 3 3 9}$ & 0.336 & 0.246 & 0.754 \\
\hline Temp & 0.007 & 0.102 & -0.842 & 0.720 & 0.280 \\
\hline Enso & 0.027 & 0.066 & 0.892 & 0.692 & 0.308 \\
\hline Season & 0.073 & 0.159 & -0.785 & 0.646 & 0.354 \\
\hline Year & -0.016 & 0.189 & 0.746 & 0.592 & 0.408 \\
\hline Eigenvalue & 3.74 & 2.97 & 2.58 & & \\
\hline$\%$ total variance & 24.9 & 19.8 & 17.18 & & \\
\hline Accumulative variance & 24.9 & 44.7 & 61.9 & & \\
\hline
\end{tabular}

Predictor variables: Year $=$ sampling year, Season $=$ climatic season $($ dry/rainy $)$, Temp $=$ surface temperature values, Enso $=$ Multivariate El Niño index, Size $=$ host body size (total length), $K_{n}=$ condition factor, Diet $=$ host diet variety, EndoR $=$ number of endoparasite species, EctoR $=$ number of ectoparasite species, EndoT $=$ total number of endoparasite per host, EctoT $=$ total number of ectoparasite per host. Infracommunity parameters: Richness $=$ number of parasite species per infracommunity, Diversity $=$ Brillouin diversity index values, Load $=$ total number of parasites per infracommunity, Evenness $=$ species evenness.

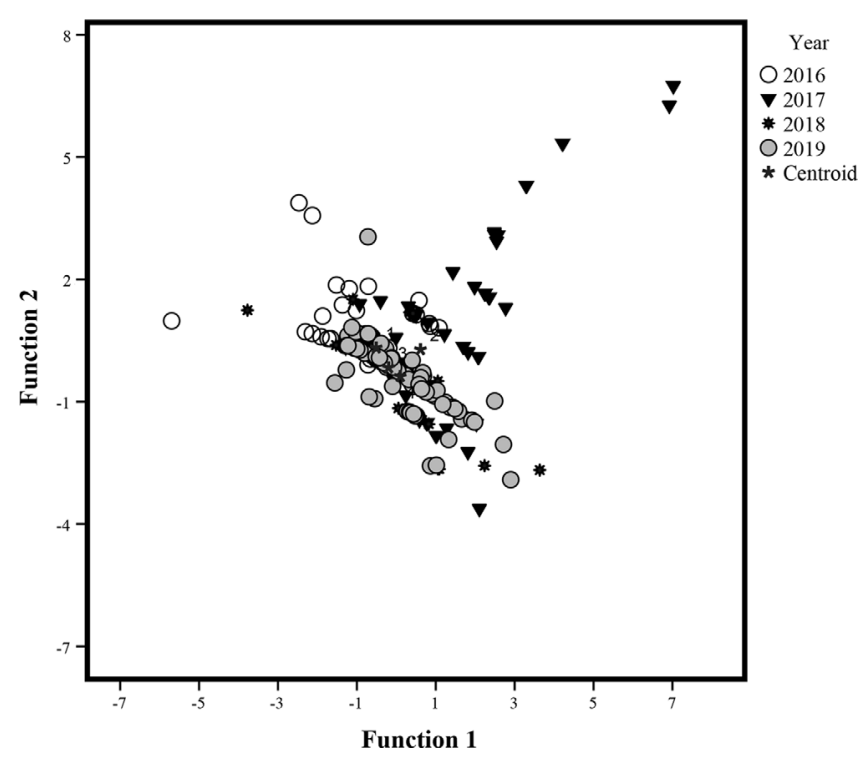

Figure 4. Graphic of multivariate discriminant analyses for parasite communities of Caranx sexfasciatus. The symbols represent each one of the fish examined in each sampling year. Centroid = mean group.

dispersion pattern of parasites in marine fish [3, 18, 37]. However, the widely variable aggregation levels of five helminth species (the monogeneans N. pacifica and Pr. manteri; the digeneans B. varicus and Ectenurus virgulus; and the nematode Anisakis sp.) suggest the existence of differences in host exposure rates to parasites. Differing exposure rates could be due to the influence of local environmental factors [35], or the differences in host feeding behavior revealed in the diet analysis. In contrast, some contact-transmitted parasites such as monogeneans, exhibit high host specificity and can be more abundant in larger fish that form schools [23, 29], like C. sexfasciatus. Some studies also suggest that DI values can be influenced by sample size $[33,35]$, but there was no positive correlation between DI values and sample sizes in the present analysis.

\section{Component communities}

The parasite communities in $C$. sexfasciatus exhibited similar patterns at the component and infracommunity levels: low species numbers (2.22-2.96 species on average per host), low species diversity, and dominance by a single species (the monogenean Pr. manteri, Table 2). Species richness at the component level (8-25 species, Table 2) is similar to that reported previously in other Caranx species in the Americas, such as C. hippos (19 species), C. latus (17 species), and C. caballus (18 species) $[15,21]$. However, overall parasite fauna of $C$. sexfasciatus included 32 species across the four sampling years. The broad geographical distribution of C. sexfasciatus may explain this greater overall richness since 
Table 4. Discriminant analysis classification showing the numbers and percentages of the Caranx sexfasciatus classified in each sampling year (rows correspond to group memberships). The bottom of the table shows the matrix of classification coefficient values for parasites that allowed differentiation among sampling years; coefficient values in bold indicate the importance of each species of parasite in distinguishing among years.

\begin{tabular}{lccccc}
\hline Sampling year & 2016 & 2017 & 2018 & 2019 & 10 \\
\hline 2016 & 22 & 7 & 57 & 28 & 22.9 \\
2017 & 5 & 22 & 81 & 42 & 76.4 \\
2018 & 3 & 3 & 53 & & \\
2019 & 8 & 5 & 0.063 & 0.068 \\
Parasite & & 0.030 & 0.024 & $\mathbf{0 . 0 4 8}$ \\
$\quad$ Protomicrocotyle manteri & $\mathbf{0 . 1 3 5}$ & 0.044 & 0.056 & 0.032 \\
$\quad$ Neomicrocotyle pacifica & 0.021 & 0.112 & 0.052 & 0.089 \\
$\quad$ Ectenurus virgulus & $\mathbf{0 . 3 4 9}$ & $\mathbf{1 . 2 3 9}$ & $\mathbf{0 . 9 8 2}$ & $\mathbf{1 . 3 8 9}$ \\
$\quad$ Caligus alalongae & 0.214 & 0.961 & & \\
Lernanthropus ilishae & -0.070 & &
\end{tabular}

hosts with a broad geographical distribution are exposed to large numbers of parasite species by interaction with myriad intermediate host species throughout their distribution range [40]. Species composition similarity between the parasite communities (Fig. 2) was considered generally low at both levels (qualitative and quantitative levels: $<65 \%$ ), compared to the similarity recorded for parasite communities of other marine fish examined on the Mexican Pacific coast, such as Caranx caballus 78.5\% [14], Oligoplites altus 85\% [39], or Parapsettus panamensis $76 \%$ [51]. This low similarity can be attributed to the fact that $47 \%$ (15 species: six ectoparasites and nine endoparasites) of the identified parasite species occurred only in one or two sampling years (Table 1). This suggests that annual variations in biotic (e.g. diet, body size, availability of larva-infected prey) and/or abiotic environmental factors $[14,23,39,49,51]$, may have been responsible for the low similarity between the parasite communities of $C$. sexfasciatus observed in the present study.

\section{Infracommunities}

Several biotic and abiotic factors are known to strongly influence species richness and diversity in the parasite communities of marine fish over time [14, 23, 34, 39, 48, 51]. The PCA results (Fig. 3) indicated that infracommunity species richness and diversity depended heavily on the occurrence of a distinctive set of component species (both ectoand endoparasite species). However, these component species' frequency or abundance varied between sampling years (Table 1). Temporal variations in parasite infracommunity structure and species composition have been attributed to variations in the prevalence and/or abundance of some dominant taxa [4, $14,16,38,39,51]$. As the ICI results suggest, the ectoparasites $N$. pacifica, $\mathrm{Pr}$. manteri, and $\mathrm{Ca}$. robustus play an important role in structuring the parasite infracommunities of $C$. sexfasciatus. Due to ease of transmission through direct contact, ectoparasite populations contribute substantially to the structuring of infracommunities in gregarious fish such as carangids [23, 29, 31]. Fluctuations in the infection levels of some endoparasite species (e.g. B. varicus and the Tetraphyllidean cestode) can be attributed mainly to changes in $C$. sexfasciatus diet or foraging habitat, both between sampling years and climatic seasons (dry/rainy). Host body size was an important predictor of the total parasite load, as suggest by the global positive correlation $(r=0.633)$ recorded for this variable in the PCA (Table 3). In marine fish, body size has proved to be the main predictor of total parasite abundance [23, 39, 40]. A large body size can facilitate parasite colonization, since larger individuals tend to ingest greater quantities of food and are older and thus have had more time to accumulate parasites than smaller individuals $[39,40]$. However, the negative correlations registered between host body size and species richness and diversity parameters suggest that the richest and most diverse parasite infracommunities were recorded in smaller rather than larger fish. Ontogenic changes in the feeding habits [10, 11, 20, 51] of C. sexfasciatus can explain the broad dietary diversity in smaller fish (for example, October 2016 and November 2018 samples, Table 2). This would in turn raise infracommunity species richness and diversity in these individuals (Table 2), as indicated in the diet analysis. Parasites are known to be useful as biological tags in distinguishing between fish stocks of the same species [47, 54], but the parasite fauna method has rarely been used to quantify possible variations in community structure and species composition over time. The discriminant analysis results (Table 4) indicated that the high variation in the infection levels of at least five parasite species (the monogeneans N. pacifica and Pr. manteri; the digenean E. virgulus; and the copepods $C a$. alalongae and L. ilishae) may generate substantial changes in the parasite community structure of $C$. sexfasciatus over time. In other words, even though community species composition remains relatively stable, its structure may be less predictable.

Overall, the parasite communities of $C$. sexfasciatus were characterized by high numerical dominance of ectoparasites, mainly monogenean species. Community structure and species composition varied between sampling years and climatic seasons. Despite occurrence of a distinctive set of host-specialist parasites (monogenean species), similarity between the component parasite communities was generally low. Seasonal or local variations in some biotic (e.g. feeding behavior, body size, and infected prey availability) and abiotic environmental factors are possible sources of the observed interannual variations in C. sexfasciatus parasite community structure and species composition.

Acknowledgements. The authors thank the fishermen who caught the fish at each sampling date, as well as the students of the Marine 
Ecology Academic Unit (UAGro) for their assistance with field and laboratory work. Four anonymous reviewers provided useful comments that substantially improved this manuscript.

\section{References}

1. Aguirre-Macedo ML, Vidal-Martínez VM, González-Solís D, Caballero-Pinzon PI. 2007. Helminth communities of four commercially important fish species from Chetumal Bay, Mexico. Journal of Helminthology, 81(1), 19-31.

2. Allen GR, Robertson DR. 1998. Fishes of the tropical eastern Pacific. Honolulu, Hawaii: University of Hawaii Press. p. 327.

3. Amarante CF, Tassinari WS, Luque JL, Pereira MJS. 2015. Factors associated with parasite aggregation levels in fishes from Brazil. Brazilian Journal of Veterinary Parasitology, 24, 174-182.

4. Balboa L, George-Nascimento M. 1998. Variaciones ontogenéticas y entre años en las infracomunidades de parásitos metazoos de dos especies de peces marinos de Chile. Revista Chilena De Historia Natural, 71, 27-37.

5. Bautista-Hernández CE, Monks S, Pulido-Flores G. 2013. Los parásitos y el estudio de su biodiversidad: un enfoque sobre los estimadores de la riqueza de especies, in Estudios científicos en el estado de Hidalgo y zonas aledañas, Pulido-Flores G, Monks S, Editors. Zea Books: Lincoln, Nebraska. p. 13-17.

6. Bautista-Hernández CE, Monks S, Pulido-Flores G, RodríguezIbarra AE. 2015. Revisión bibliográfica de algunos términos ecológicos usados en parasitología, y su aplicación en estudios de caso, in Estudios en Biodiversidad, Pulido-Flores G, Monks S, López-Herrera M, Editors. Zea Books: Lincoln, Nebraska. p. 11-19.

7. Bush AO, Aho JM, Kennedy CR. 1990. Ecological versus phylogenetic determinants of helminth parasite community richness. Evolutionary Ecology, 4(1), 1-20.

8. Bush AO, Lafferty KD, Lotz JM, Shostak AW. 1997. Parasitology meets ecology on its own terms: Margolis et al. revisited. Journal of Parasitology, 83(4), 575-583.

9. Bush AO, Fernandez JC, Esch GW, Seed JR. 2001. Parasitism: the diversity and ecology of animal parasites. Cambridge, UK: Cambridge University Press. p. 729.

10. Campos CM, Takemoto RM, Fonseca VE, Moraes FR. 2009. Ecology of the parasitic endohelminth community of Piaractus mesopotamicus (Holmberg, 1887) (Characiformes) from Aquidauana and Miranda Rivers, Pantanal, state of Mato Grosso do Sul, Brazil. Brazilian Journal of Biology, 69, 87-92.

11. Cotta-Ribeiro T, Molina-Ureña H. 2009. Ontogenic changes in the feeding habits of the fishes Agonostomus monticola (Mugilidae) and Brycon behreae (Characidae), Térraba River, Costa Rica. Revista de Biologia Tropical, 57, 285-290.

12. Díaz F, George-Nascimineto M. 2002. Estabilidad temporal de las infracomunidades de parásitos en la borrachilla Scartichthys viridis (Valenciennes, 1836) (Pisces: Blenniidae) en la costa central de Chile. Revista Chilena De Historia Natural, 75, 641-649.

13. Froese R. 2006. Cube law, condition factor and weight-length relationships: history, meta-analysis and recommendations. Journal of Applied Ichthyology, 22, 241-253.

14. Gallegos-Navarro Y, Violante-González J, Monks S, GarcíaIbáñez S, Rojas-Herrera AA, Pulido-Flores G, Rosas-Acevedo JL. 2018. Factors linked to temporal and spatial variation in the metazoan parasite communities of green jack Caranx caballus (Günther 1868) (Pisces: Carangidae) from the Pacific coast of Mexico. Journal of Natural History, 52(39-40), 2573-2590.
15. Gallegos-Navarro Y, Violante-González J, Monks S, GarcíaIbáñez S, Rojas-Herrera AA, Rosas-Acevedo JL. 2019. Metazoan parasite communities of Selar crumenophthalmus and Decapterus muroadsi (Pisces: Carangidae) from Mexican Pacific coasts. New Zealand Journal of Marine and Freshwater Research, 53(3), 377-396.

16. Garcias F, Mendoza R, George-Nascimento M. 2001. Variacion entre anos de las infracomunidades de parasitos metazoos de la corvina Cilus gilberti (pices: Sciaenidae) en Chile. Revista Chilena de Historia Natural, 74, 833-840.

17. Henriquez V, Gonxalez MT. 2012. Patterns of variation in parasite component communities and infracommunities of a littoral fish species from the northern coast of Chile. Journal of Helminthology, 88, 88-96.

18. Iannacone J, Moron L, Guizado S. 2010. Between-year variation of metazoan parasite fauna on Sciaena deliciosa (Tschudi, 1846) (Perciformes: Sciaenidae) in Lima, Peru. Latin American Journal of Aquatic Research, 38(2), 218-226.

19. Lima-Junior SE, Goltein R. 2001. A new method for the analysis of fish stomach contents. Maringá, 23(2), 421-424.

20. Luque JL, Chaves ND. 1999. Ecologia da comunidade de metazoários parasitos de Pomatomus saltator (Osteichthyes, Pomatomidae) do litoral do Estado do Rio de Janeiro. Revista Brasileira de Zoologia, 16(3), 711-723.

21. Luque JL, Ramos Alves D. 2001. Ecologia das comunidades de metazoários parasitos, do xaréu, Caranx hippos (Linnaeus) e do xerelete, Caranx latus Agassiz (Osteichthyes, Carangidae) do litoral do estado do Rio de Janiro, Brasil. Revista Brasileira de Zoologia, 18(2), 399-410.

22. Luque JL, Tavares LE. 2007. Checklist of Copepoda associated with fishes from Brazil. Zootaxa, 1579, 1-39.

23. Luque JL, Mouillot D, Poulin R. 2004. Parasite biodiversity and its determinants in coastal marine teleost fishes of Brazil. Parasitology, 128(6), 671-682.

24. Machado MH, Pavanelli GC, Takemoto RM. 1995. Influence of the type of environment and of the hydrological level variation in endoparasitic infrapopulations of Pseudoplatystoma corruscans (Agassiz) and Schizodon borelli (Boulenger) (Osteichthyes) of the high Paraná River. Revista Brasileira de Biologia, 12(4), 961-976.

25. Magurran AE. 2004. Measuring biological diversity. Malden, MA: Blackwell Publishing Company. p. 256.

26. Marcogliese DJ. 2002. Food webs and the transmission of parasites to marine fish. Parasitology, 124(7), 83-89.

27. Mendlova M, Simkova A. 2014. Evolution of host specificity in monogeneans parasitizing African cichlid fish. Parasites and Vectors, 7(69), 1-14.

28. Morales-Serna FN, Pinacho-Pinacho CD, Gómez S, PérezPonce de León G. 2014. Diversity of sea lice (Copepoda: Caligidae) parasitic on marine fishes with commercial and aquaculture importance in Chamela Bay, Pacific coast of Mexico by using morphology and DNA barcoding, with description of a new species of Caligus. Parasitology International, 63(1), 69-79.

29. Morand S, Poulin R. 1998. Density, body mass and parasite species richness of terrestrial mammals. Evolutionary Ecology, $12,717-727$.

30. Muñoz G, Grutter AS, Cribb TH. 2006. Endoparasite communities of five fish species (Labridae: Cheilininae) from Lizard Island: how important is the ecology and phylogeny of the hosts? Parasitology, 132(3), 363-374.

31. Palacios-Fuentes P, Landela MF, Gonzalez MT, Plaza G, Ojeda FP, Muñoz G. 2015. Is ectoparasite burden related to host density? Evidence from nearshore fish larvae off the coast of central Chile. Aquatic Ecology, 49(1), 91-98. 
32. Pérez-Ponce de León G, García-Prieto L, Mendoza-Garfias B, León-Règagnon V, Pulido-Flores G, Aranda-Cruz C, GarcíaVargas F. 1999. Listados faunísticos de México. IX. Biodiversidad de helmintos parásitos de peces marinos y estuarinos de la Bahía de Chamela, Jalisco, in Listados faunísticos de México, García-Aldrete AN, Editors. Vol. IX. Universidad Nacional Autonoma de México, Instituto de Biologia: Distrito Federal, México. p. 1-51.

33. Poulin R. 1993. The disparity between observed and uniform distributions: a new look at parasite aggregation. International Journal of Parasitology, 23(7), 937-944.

34. Poulin R. 1995. Phylogeny, ecology and the richness of parasite communities in vertebrates. Ecological Monographs, 65, 283-302.

35. Poulin R. 2013. Explaining variability in parasite aggregation levels among host samples. Parasitology, 140(4), 541-546.

36. Poulin R, Kamiya T. 2015. Parasites as biological tags of fish stocks: a meta-analysis of their discriminatory power. Parasitology, 142, 145-155.

37. Rohde K. 2005. Marine Parasitology. Clayton, Australia: CSIRO Publishing. p. 592

38. Santana-Piñeros AM, Pech D, Vidal-Martínez VM. 2012. Spatial structure of the helminth parasite communities of the tonguefish, Symphurus plagiusa, from the Campeche coast, southern Mexico. International Journal for Parasitology, 42(10), 911-920.

39. Santos-Bustos NG, Violante-González J, Monks S, RojasHerrera AA, García-Ibáñez S, Flores-Rodríguez P, AlmazánNúñez RC, Moreno-Díaz G. 2018. Species richness and similarity of metazoan parasite communities in three species of leatherjacket (Oligoplites: Pisces: Carangidae) from the Pacific coast of Mexico. Invertebrate Biology, 137(3), 205-220.

40. Sasal P, Morand S, Guégan J-F. 1997. Determinants of parasite species richness in Mediterranean marine fishes. Marine Ecology Progress Series, 149, 61-71.

41. Sasal P, Desdevises Y, Morand S. 1998. Host-specialization and species diversity in fish parasites: phylogenetic conservatism? Ecography, 21, 639-643.

42. Sasal P, Trouvé S, Müller-Graf C, Morand S. 1999. Specificity and host predictability: a comparative analysis among monogenean parasites of fish. Journal of Animal Ecology, 68(3), 437-444.

43. Soares IA, Vieira FM, Luque JL. 2014. Parasite community of Pagrus pagrus (Sparidae) from Rio de Janeiro, Brazil: evidence of temporal stability. Brazilian Journal of Veterinary Parasitology, 23(2), 216-223.

44. Sokol RR, Rohlf FJ. 1998. Biometry, 2nd edn. San Francisco, California: W. H. Freeman and Co. p. 859.

45. Soler-Jimenez LC, Morales-Serna FN, Aguirre-Macedo ML, McLaughlin JP, Jaramillo AG, Shaw JC, James AK, Hechinger
RF, Kuris AM, Lafferty KD, Vidal-Martínez VM. 2019. Parasitic copepods (Crustacea, Hexanauplia) on fishes from the lagoon flats of Palmyra Atoll, Central Pacific. ZooKeys, 833, 85-106.

46. Tavares LER, Luque JL. 2008. Similarity between metazoan parasite communities of two sympatric brackish fish species from Brazil. Journal of Parasitology, 94, 985-989.

47. Timi JT. 2007. Parasites as biological tags for stock discrimination in marine fish from South American Atlantic waters. Journal of Helminthology, 81(2), 107-111.

48. Timi JT, Poulin R. 2003. Parasite community structure within and across host populations of a marine pelagic fish: how repeatable is it? International Journal for Parasitology, 33(12), $1353-1362$.

49. Timi J, Lanfranchi A, Luque J. 2010. Similarity in parasite communities of the teleost fish Pinguipes brasilianus in the southwestern Atlantic: infracommunities as a tool to detect geographical patterns. International Journal for Parasitology, 40, 243-254.

50. Vidal-Martínez VM, Poulin R. 2003. Spatial and temporal repeatability in parasite community structure of tropical fish hosts. Parasitology, 127, 387-398.

51. Villalba-Vasquez PJ, Violante-González J, Monks S, MarinoRomero JU, Ibáñez SG, Rojas-Herrera AA, Flores-Garza R, Rosas-Guerrero V. 2018. Temporal and spatial variations in the metazoan parasite communities of the Panama spadefish, Parapsettus panamensis (Pisces: Ephippidae), from the Pacific coast of Mexico. Invertebrate Biology, 137(4), 339-354.

52. Violante-González J, Mendoza-Franco EF, Rojas-Herrera A, Gil Guerrero S. 2010. Factors determining parasite community richness and species composition in black snook Centropomus nigrescens (Centropomidae) from Coastal Lagoons in Guerrero, Mexico. Parasitology Research, 107(11), 59-66.

53. Violante-González J, Rojas-Herrera AA, Pulido-Flores G, Monks S, Gil-Guerrero JS, Rosas-Acevedo JL, CarbajalViolante J. 2014. Influencia de las condiciones ambientales en la composición de la parasitofauna de peces en la Laguna de Tres Palos, Guerrero, México, in Calidad ambiental y desarrollo sustentable: Tomo III. Sampedro-Rosas ML, Rosas-Acevedo JL, Juárez-López AL, Editors. Universidad Autónoma de Guerrero y INDAUTOR: Ciudad de México, D.F. p. 88-120.

54. Williams HH, MacKenzie K, McCarthy AM. 1992. Parasites as biological indicators of the population biology, migrations, diet, and phylogenetics of fish. Reviews in Fish Biology and Fisheries, 2(2), 144-176.

55. Zander CD. 2004. Four-year monitoring of parasite communities in gobiid fishes of the south-western Baltic. II. Infracommunity. Parasitology Research, 93(1), 17-29.

Cite this article as: Violante-González J, Monks S, Gallegos-Navarro Y, Santos-Bustos NG, Villalba-Vasquez PJ, Padilla-Serrato JG \& Pulido-Flores G. 2020. Interannual variation in the metazoan parasite communities of bigeye trevally Caranx sexfasciatus (Pisces, Carangidae). Parasite 27, 6. 
Reviews, articles and short notes may be submitted. Fields include, but are not limited to: general, medical and veterinary parasitology; morphology, including ultrastructure; parasite systematics, including entomology, acarology, helminthology and protistology, and molecular analyses; molecular biology and biochemistry; immunology of parasitic diseases; host-parasite relationships; ecology and life history of parasites; epidemiology; therapeutics; new diagnostic tools.

All papers in Parasite are published in English. Manuscripts should have a broad interest and must not have been published or submitted elsewhere. No limit is imposed on the length of manuscripts.

Parasite (open-access) continues Parasite (print and online editions, 1994-2012) and Annales de Parasitologie Humaine et Comparée (1923-1993) and is the official journal of the Société Française de Parasitologie. 